\title{
FLORISTIC SURVEY OF FLOWERING PLANTS IN A TROPICAL COASTAL eCosystem in Veracruz, Mexico
}

\author{
Cristian Adrian Martínez-Adriano, Armando Aguirre-Jaimesª and Cecilia Díaz-Castelazo \\ Red de Interacciones Multitróficas, Instituto de Ecología A.C. Xalapa. Veracruz, México \\ 'Corresponding author: armando.aguirre@inecol.mx
}

\begin{abstract}
Mexico is a megadiverse country and Veracruz is one of the states with the highest floristic diversity. The reserve of La Mancha in central coastal Veracruz comprises several vegetation types. In this site we conducted for a year, monthly surveys of flowering or fruiting plants in six vegetation types, considering the frequency of occurrence of different species. We found a total of 147 species (131 genera and 63 families). The families most represented were Asteraceae, Fabaceae and Verbenaceae. In addition, we found nine species that had not been reported in previous floristic studies. The highest taxonomic richness was observed in the tropical deciduous flood forest with wetland (59 species), while the highest diversity was found in tropical deciduous forest $(\mathrm{SDI}=0.9562)$. We also found that the most similar vegetation types based on the composition and frequency of species were, coastal dune scrub, tropical deciduous forest and pioneer dune vegetation. The dominant plant biological form in La Mancha were herbs $(40.41 \%)$ followed by shrubs $(23.97 \%)$, vines-lianas $(18.49 \%)$ and trees $(17.12 \%)$. This study is the first to consider the frequency-occurrence of flowering and fruiting plants in La Mancha, covering six vegetation types through one year. The more relevant results of this study show the diversity of plants in different environments using the frequency of each species based on reproductive events. This information could be useful in future researches that evaluate plant-animal interactions, like pollination and seed dispersal.
\end{abstract}

Key words: floristic, flowering plants, fruiting plants, La Mancha, Veracruz.

Resumen: México es considerado un país megadiverso y Veracruz es uno de los estados con mayor diversidad florística del país. La reserva de La Mancha en la costa central de Veracruz alberga varios tipos de vegetación. En este sitio realizamos durante un año censos mensuales de la vegetación en floración o fructificación en seis tipos de vegetación, considerando la frecuencia de aparición de las diferentes especies de plantas. Encontramos en total 147 especies (131 géneros y 63 familias). Las familias mejor representadas fueron Asteraceae, Fabaceae y Verbenaceae. Además de nueve especies que no habían sido reportadas en estudios florísticos previos. La mayor riqueza taxonómica se observó en la selva baja inundable y el humedal (59 especies), mientras que, la mayor diversidad se encontró en la selva baja caducifolia $(\mathrm{SDI}=0.9562)$. Además encontramos que los tipos vegetación más similares con base a la composición y frecuencia de especies son el matorral de dunas costeras, la selva baja caducifolia y la vegetación pionera de dunas. La forma biológica predominante en La Mancha fueron las hierbas (40.41\%), seguida por arbustos (23.97\%), bejucos-lianas $(18.49 \%)$ y árboles $(17.12 \%)$. Este trabajo es el primero que analiza la frecuencia-ocurrencia de plantas con flor y/o fruto en La Mancha, abarcando seis tipos de vegetación. Los resultados más destacados muestran la diversidad de plantas en diferentes ambientes utilizando la frecuencia de cada especie durante sus eventos reproductivos. Esta información puede ser útil en futuras investigaciones sobre interacciones planta-animal, como polinización y dispersión de semillas.

Palabras clave: florística, La Mancha, plantas en floración, plantas en fructificación, Veracruz.

$M$ exico is known to be megadiverse country because it comprises approximately $10 \%$ of all existent species in the world (Mittermeier et al., 1997; Ceballos, 2007; Sarukhán et al., 2012; Espinosa-Jiménez et al., 2014). This high diversity is due to topography and complex geological structure, and the confluence of Nearctic and Neotropical regions favoring thus, a high variety of climates, soils, fauna and vegetation types, ranging from evergreen tropical forest to arid ecosystems (Miranda and Hernández, 1963; NeyraGonzález and Durand-Smith, 1998; González-Medrano, 2003; Rzedowski, 2006; Espinosa-Jiménez et al., 2014). These characteristics results in high flora diversity, estimat- 
ed in 36,000 plant species for Mexico (Neyra-González and Durand-Smith, 1998), concentrated in Chiapas, Oaxaca and Veracruz mainly; further, the arid ecosystems comprise a great proportion of endemic species (Rzedowski, 1991; 1993).

In Mexico, Veracruz State is the third place in floristic richness with 7,855 taxa only below of Chiapas and Oaxaca (Rzedowski, 1991; 1993; Castillo-Campos et al., 2011); such richness is distributed in 19 vegetation types (Miranda and Hernández, 1963). La Mancha reserve is located in the central coast of Veracruz, where several vegetation types are represented (Castillo-Campos and Travieso-Bello, 2006). In this area several floristic surveys has been developed focused on biodiversity, ecological and management aspects. Many of this studies are related on characterization of biological forms, or on inventories on particular vegetation associations (Moreno-Casasola et al., 1982; García-Franco, 1996; Castillo and Moreno-Casasola, 1998; Castillo-Campos and Medina-Abreo, 2005; Moreno-Casasola and Travieso-Bello, 2006; Peralta-Peláez and Moreno-Casasola, 2009; MorenoCasasola et al., 2010; Infante-Mata et al., 2011); other studies, are more exhaustive vegetation compendia on coastal area (Novelo-Retana, 1978; Castillo-Campos and TraviesoBello, 2006).

Coastal vegetation in La Mancha is the result of an interphase between the sea and land, where their ecological processes are linked (Fragoso, 1995) to configure complex ecosystems; it is particularly interesting because it is so far known the only site in México that house tropical sub-deciduous and tropical dry forest occurring over coastal sand dunes (Castillo-Campos, 2006). Also due to dune formation, sand movement, dune erosion and marked seasonal abiotic changes (Moreno-Casasola, 1982; Moreno-Casasola and Travieso-Bello, 2006; Rico-Gray et al., 2012; SánchezGalván et al., 2012), thus generating an local ecological scenario where a great diversity of biological processes can be developed, such as pollination, seed dispersal, recruitment, competition, migration and herbivory (Turner et al., 1996; Benítez-Malvido, 1998; Benítez-Malvido et al., 1999; Cordeiro and Howe, 2001; Wright and Duber, 2001; Chacoff et al., 2004).

In this area previous studies has been developed only for a few specific plant species, also studies at community level are scarce, and the available studies are centered on reproductive biology (Rico-Gray and Castro, 1996; GarcíaFranco and Rico-Gray, 1997a; 1997b; Arceo-Gómez et al., 2012), ant-plant interactions (Rico-Gray et al., 1998; Torres-Hernández et al., 2000; Cuautle and Rico-Gray, 2003), diversity of ants by vegetation types (Rojas et al., 2014), floral visitors richness (González-Vanegas, 2011; RodríguezMorales et al., 2013), and frugivory by birds (Ortiz-Pulido, 1997). In addition, community-level studies have been developed for extrafloral-nectary bearing plants (Rico-Gray, 1993; Díaz-Castelazo et al., 2004; Rico-Gray et al., 2012), pollination networks (Hernández-Yáñez et al., 2013), and also studies on phenology (Castillo and Carabias, 1982; Mehltreter, 2006).

Despite the wide range of studies that have been conducted in La Mancha there are not investigations with a focus at the level of the plant community and further considering reproductive effort. Based on this background we did the characterization of flowering and fruiting plants, and show how is the intensity of these phenological stages over a year, including different vegetation types inside La Mancha. The objectives of this study were: (a) make a floristic characterization of the plant flowering and fruiting species in six vegetation types, (b) determine the biological forms of flowering plants, and (c) evaluate the reproductive offer (occurrence-frequency) in these vegetation types.

\section{Materials and methods}

Study area. Field work was carried out at Centro de Investigaciones Costeras La Mancha (CICOLMA) located on the coast of Veracruz, Mexico (96 24'48'W, 1940'33'N and $\left.96^{\circ} 22^{\prime} 25^{\prime \prime} \mathrm{W}, 19^{\circ} 31^{\prime} 49^{\prime \prime} \mathrm{N}\right)$. The CICOLMA is a natural reserve area managed by Instituto de Ecología, A.C. (INECOL) and comprises an area of 83.29 ha, which includes several native vegetation types (including wetlands and dune vegetation), transformed forest/agricultural sites, experimental areas and a field station (Moreno-Casasola and Monroy, 2006). The CICOLMA was declared as Ramsar site in 2004 and the protection of natural resources and wildlife is given to INECOL by Mexican federal regulatory organisms in the matter, framed by intergovernmental treaty "The Ramsar Convention on Wetlands" (Secretaría de la Convención de Ramsar, 2013).

The main vegetation types in La Mancha are tropical sub-deciduous forest on two soil types based on mineralogical and physicochemical analyses (Dubroeucq et al., 1992; Geissert and Dubroeucq, 1995), tropical deciduous forest, sand dune scrub, mangrove forest, freshwater marsh and tropical deciduous flood forest (Miranda and Hernández, 1963; Rico-Gray 1993; Castillo-Campos and Travieso-Bello, 2006). The climate is warm sub-humid, and the annual precipitation average is $1,300 \mathrm{~mm}, 78 \%$ of the total annual precipitation occurs during the rainy season, the mean annual temperature is $25^{\circ} \mathrm{C}$ (Moreno-Casasola et al., 1982; Kellman, 1990; Kellman and Roulet, 1990; Kavanagh and Kellman, 1992; Kellman and Delfosse, 1993; Rico-Gray, 1993; Díaz-Castelazo et al., 2004), $18{ }^{\circ} \mathrm{C}$ in coldest month and $>22{ }^{\circ} \mathrm{C}$ on warmest month (Travieso-Bello and Campos, 2006). The study site is markedly seasonal (Rico-Gray and Oliveira, 2007; Díaz-Castelazo et al., 2010) with three distinctively different seasons, the dry season (FebruaryMay), the rainy season (June-September), the winter cold front season ("nortes", October-January). In this area, 837 plant species have been recorded, $50 \%$ of which are herba- 
ceous plants and the rest are shrubs, trees and vines-lianas (Castillo-Campos and Travieso-Bello, 2006).

The study was carried out at six different vegetation types: (1) coastal dune scrub (CDS), (2) pioneer dune vegetation (PDV), 3) tropical sub-deciduous forest in young soil (TSF1), (4) tropical sub-deciduous forest in old soil (TSF-2), (5) tropical deciduous forest (TDF), and (6) tropical deciduous flood forest with wetland (TDF-W). For the present study, we unified the Gymnanthes forest with tropical sub-deciduous forest, and savanna with tropical deciduous forest, both in one vegetation type (Figure 1).

Sampling design. In each site we established one transect with 20 permanent points separated approximately $20 \mathrm{~m}$ each other (Figure 1). Based on each permanent point, we established round plots with five meters in radius from centered fixed point like Franco-Gaona et al. (1998). In each round plot we recorded all plant species in flowering and/or fruiting stages. With the species recorded by point, we calculated the presence and the occurrence frequency by species for each point.

The samplings were made monthly in all vegetation types to record all possible flowering or fruiting plants that were distributed in the area. For each plant species we recorded the vegetation type, biological forms and presence in each point, in order to obtain frequency of occurrence. In tropical sub-deciduous forest in young and old soil, tropical deciduous forest, and tropical deciduous flood forest with wetland (Figure 2), only plants under canopy were considered; thus, those species above canopy were discarded.

Botanical sampling and identification. We collected two or more samples of each species in order to carry out the botanical identification. The determination of our botanical

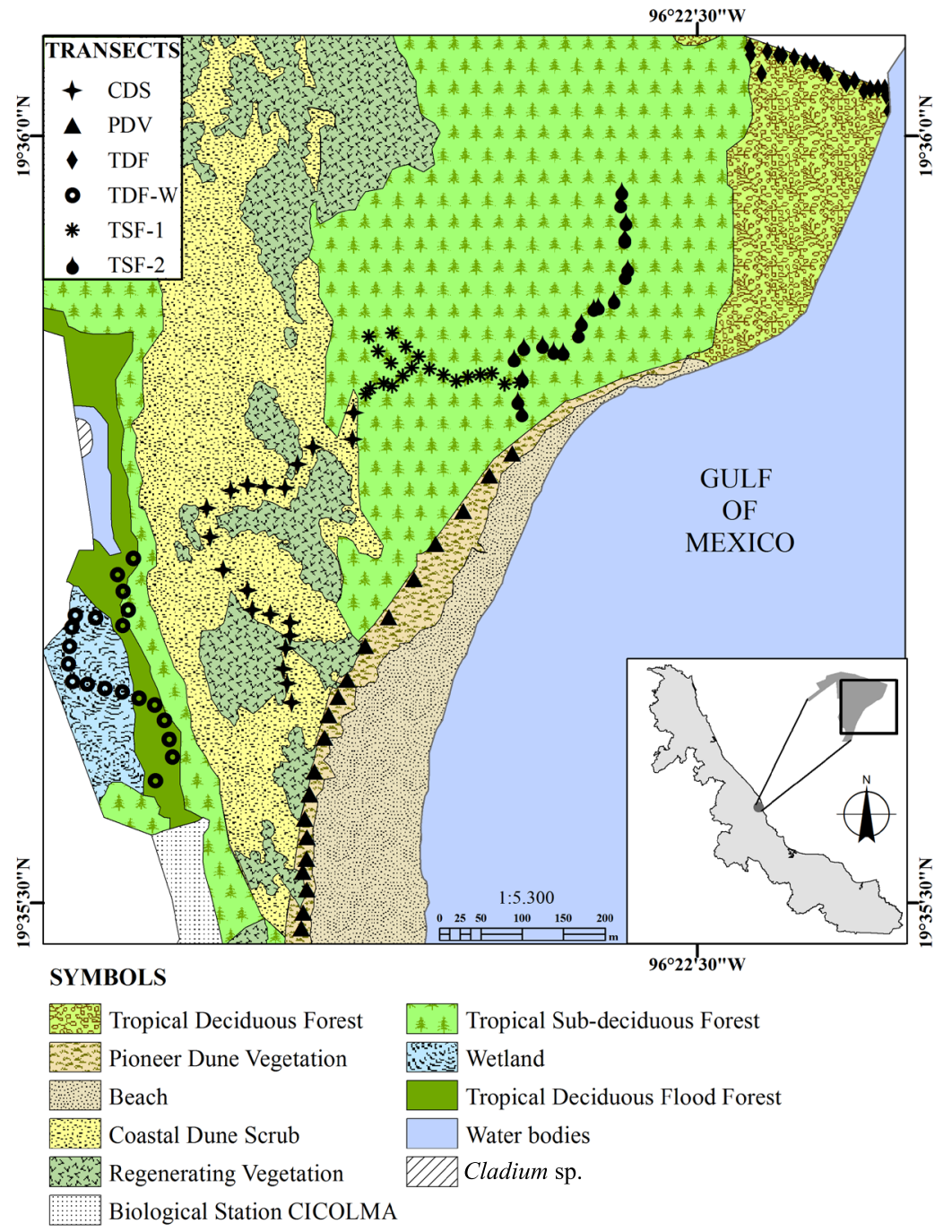

Figure 1. Location of the study area in La Mancha reserve; each symbol corresponds to sites where census was developing in the different vegetation types. (Map was actualized by Elisa Zaragoza-Quintana from Moreno-Casasola and Monroy, 2006, based on Google Earth images [June, 2012] and field observations). 

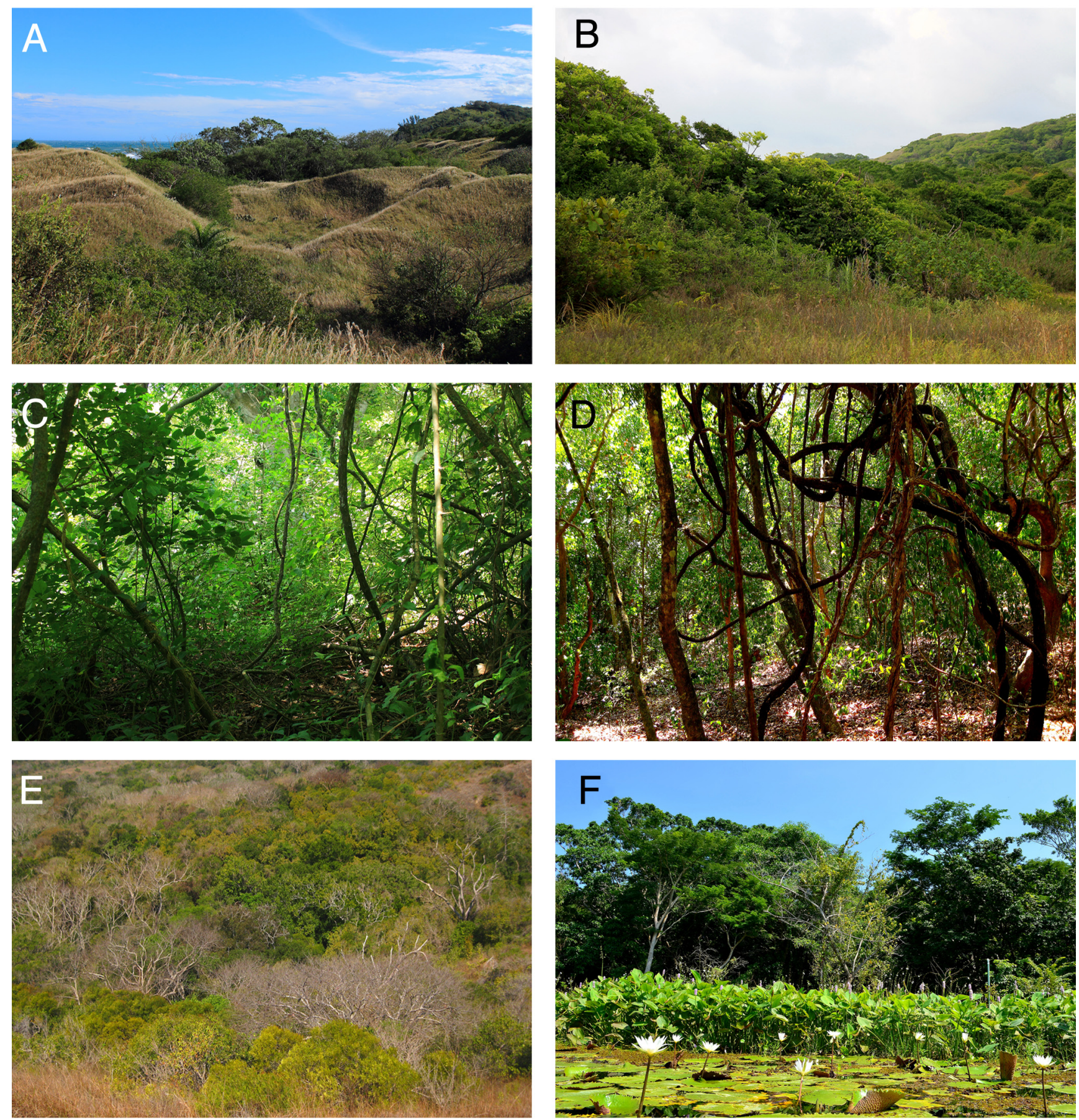

Figure 2. Landscapes showing the different vegetation types where data were collected. (A) coastal dune shrub, (B) pioneer dune vegetation, (C) tropical sub-deciduous forest in young soil, (D) tropical sub-deciduous forest in old soil, (E) tropical deciduous forest, and (F) tropical deciduous flood forest with wetland.

samples was performed by comparing them with specimens deposited in the herbarium XAL, also with the aid of taxonomic keys and field guides of the plant species of the area, as well as personally consulting with botanical specialists. The used nomenclature was according to The International Plant Names Index.
Richness estimates in sampled vegetation types. Floristic richness was expressed as the number of species present in each transect. Also, with the identity of species in the six transects we elaborated a matrix with frequency of occurrence to calculate the Simpson Dominance Index which ranges from 0 to 1 , where 0 means no dominance and 1 
means species dominance within the community (D), larger index values meaning lower diversity (Simpson, 1949;1960). With D estimates, we obtained, in order to establish habitat plant diversity, the Simpson's Diversity Index (SDI = 1 - D) with software PAST 2.01 (Hammer et al., 2001), this index ranges 0 to 1 (being most diverse the values close to 1 , and less diverse values close to 0 ). Also, we grouped the vegetation types with Jaccard Similarity Cluster Analysis to determine which vegetation types were most similar. Finally, we use EstimateS software (Colwell, 2013) to calculate the Chao-Jaccard Similarity Index from frequency of incidence (Chao et al., 2005); this is an accurate estimator because it considers species abundance or frequency of incidence.

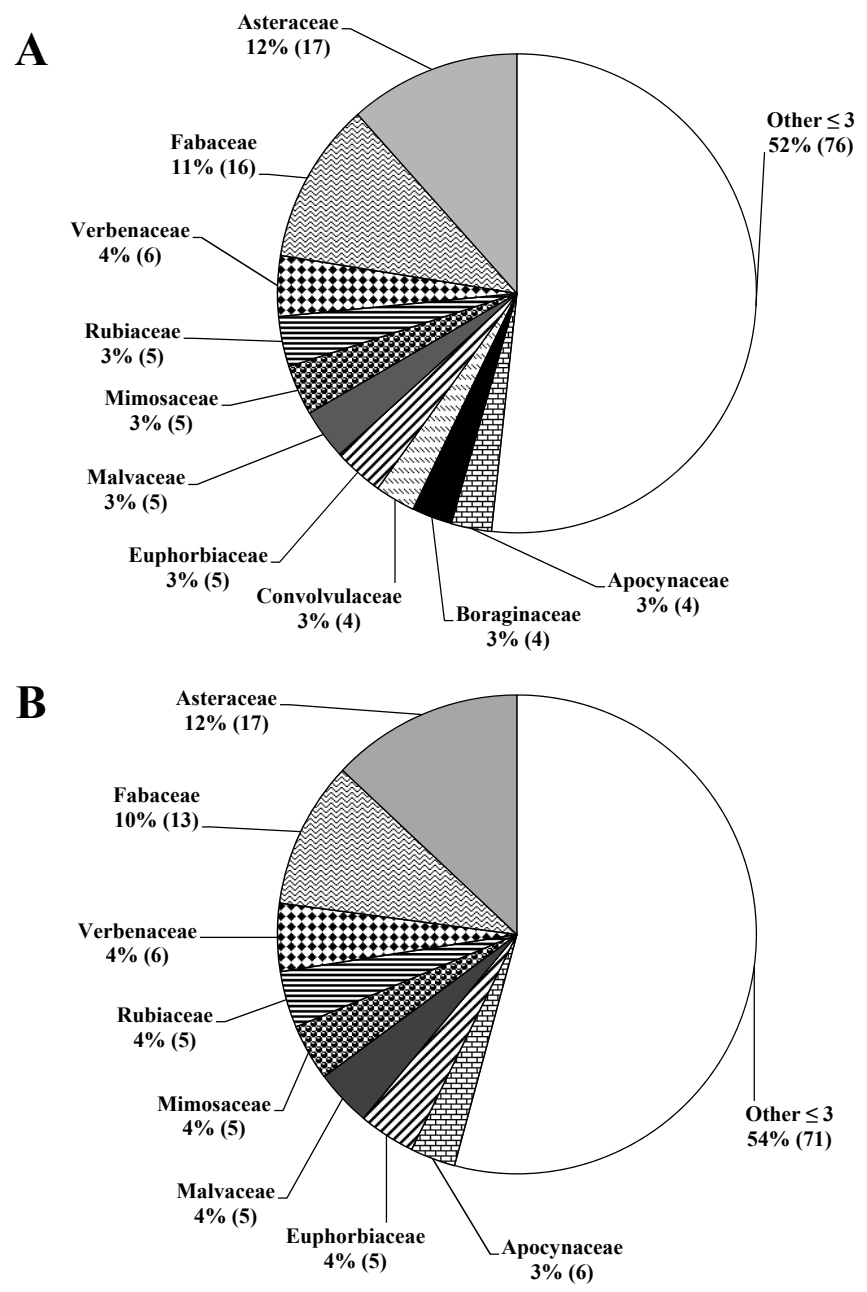

Figure 3. Taxonomic representativeness of flowering or fruiting plants in La Mancha. A) Percent of species by family, Fabaceae and Asteraceae comprised $12 \%$ of all species, while $52 \%$ correspond to taxa with less (or equal) to three species per family. B) Number of genera by family. We observe the same tendency with Fabaceae and Asteraceae comprising the major number of genera (22\%), while $54 \%$ correspond to genera with less (or equal) to two genera per family. In parentheses is show the number of species and genera respectively.

\section{Results}

Floristic richness estimates in each vegetation type. From March 2013 to May 2014 we obtained 3,564 records of plants in flowering or fruiting stage, belonging to 63 families, 131 genera, and 147 species (Table 1). Asteraceae showed the largest number of genera and species (17 and 17 respectively), followed by Fabaceae (16 species) and Verbenaceae both with six species and six genera (Figure 3A, B). These three families comprise $27 \%$ of all observed species, while the other families only had five or less species. Among the flowering and/or fruiting plant species at our sampling points, we found interesting species from a conservationist perspective, such as the threatened wetland tree species Conocarpus erectus (according to Mexican conservation regulation NOM-059-SEMARNAT-2010 [SEMARNAT, 2010]), and Chamaecrista chamaecristoides (Caesalpinaceae) and Palafoxia lindenii (Asteraceae) both as endemic species (according to Martínez and Moreno-Casasola, 1998; ÁlvarezMolina et al., 2013). In addition it is important to note that nine species not have been cited previously for this study area: Neurolaena lobata (Asteraceae), Tillandsia utriculata (Bromeliaceae), Capparidastrum frondosum (Capparaceae), Crossopetalum gaumeri (Celastraceae), Calliandra eriophylla (Mimosaceae), Desmodium scorpiurus (Fabaceae), Lonchocarpus fuscopurpureus (Fabaceae), Vigna adenantha (Fabaceae), and Trichocentrum luridum (Orchidaceae).

On the other hand, in terms of vegetation types, we found the highest taxonomic richness in tropical deciduous flood forest with wetland (59), followed by coastal dune scrub with 50 species, pioneer dune vegetation (47 species), and tropical deciduous forest (41 species). Considering families, Fabaceae had the highest contribution at tropical deciduous flood forest with wetland and coastal dune scrub. In the tropical deciduous forest the highest contribution is given by Asteraceae and Fabaceae, whereas Asteraceae was a mainly important family at pioneer dune vegetation. The species diversity index showed that the tropical deciduous forest was the most diverse (SDI $=0.9562$ ), followed by pioneer dune vegetation (SDI $=0.9437)$, coastal dune scrub (SDI $=0.9377$ ), tropical deciduous flood forest with wetland (SDI $=0.9036)$, tropical sub-deciduous forest in old soil (SDI $=0.8677$ ), and tropical sub-deciduous forest in young soil (SDI $=0.6254)$. The analysis of Jaccard Similarity formed three different clusters, the first group consisting of coastal dune scrub (CDS), tropical deciduous forest (TDF) and pioneer dune vegetation (PDV), where both the deciduous tropical forest (TDF), and pioneer dune vegetation (PDV) had the highest values of Chao-Jaccard similarity with coastal dune scrub (CDS). In turn, the second group was formed of two subtypes of tropical subdeciduous forest, while the tropical deciduous flood forest with wetland formed a separate cluster non-similar to any other vegetation type (Table 2, Figure 4). 
Table 1. Plant species, biological forms and the flowering or fruiting total occurrence frequency recorded in six vegetation types inside to La Mancha, reserve. CDS = coastal dune scrub, PDV = pioneer dune vegetation, TSF- $1=$ tropical sub-deciduous forest in young soil, TSF- $2=$ tropical sub-deciduous forest in old soil, TDF $=$ tropical deciduous forest, and TDF-W $=$ tropical deciduous flood forest with wetland. $\left({ }^{*}\right)$ denoted the new reported species and $(\mathrm{X})$ presence in vegetation types. The used nomenclature and scientific authorities was according to The International Plant Names Index.

\begin{tabular}{|c|c|c|c|c|c|c|c|c|c|}
\hline \multirow[t]{2}{*}{ FAMILY } & \multirow[t]{2}{*}{ SPECIES } & \multicolumn{6}{|c|}{ ENVIRONMENT } & \multirow{2}{*}{$\begin{array}{l}\text { BIOL. } \\
\text { FORM }\end{array}$} & \multirow[t]{2}{*}{ FREQUENCY } \\
\hline & & CDS & PDV & TSF-1 & TSF-2 & TDF & TDF-W & & \\
\hline Acanthaceae & Blechum pyramidatum (Lam.) Urb. & & & & & & $x$ & Herb & 2 \\
\hline Alismataceae & Sagittaria lancifolia L. & & & & & & $x$ & Herb & 90 \\
\hline \multirow[t]{2}{*}{ Amaranthaceae } & Achyranthes aspera L. & & & & $x$ & & & Herb & 2 \\
\hline & Iresine diffusa Humb. \& Bonpl. ex Willd. & $x$ & $x$ & & & & & Herb & 24 \\
\hline Annonaceae & Annona glabra L. & & & & & & $x$ & Tree & 3 \\
\hline Apiaceae & Hydrocotyle bonariensis Lam. & & & & & & $x$ & Herb & 6 \\
\hline \multirow[t]{5}{*}{ Apocynaceae } & $\begin{array}{l}\text { Pentalinon andrieuxii (Müll.Arg.) B.F.Hansen } \\
\text { \& Wunderlin }\end{array}$ & & & & & $x$ & & Vine & 4 \\
\hline & Pinochia peninsularis (Woodson) M.E.Endress & & & & & $x$ & & Vine & 9 \\
\hline & \& B.F.Hansen & & & & & & & & \\
\hline & Stemmadenia pubescens Benth. & & & $x$ & & & & Tree & 1 \\
\hline & Tabernaemontana alba Mill. & & & & & & $x$ & Tree & 4 \\
\hline Araceae & Spathiphyllum cochlearispathum (Liebm.) Engl. & & & & & & $x$ & Herb & 3 \\
\hline Arecaceae & Cocos nucifera L. & & $x$ & & & & & Tree & 11 \\
\hline \multirow[t]{2}{*}{ Asclepiadaceae } & Asclepias curassavica L. & & & & & & $x$ & Herb & 18 \\
\hline & $\begin{array}{l}\text { Metastelma schlechtendalii (Decne.) Standl. } \\
\text { \& Steyerm. }\end{array}$ & $\mathrm{X}$ & $\mathrm{X}$ & & & $x$ & & Vine & 49 \\
\hline \multirow[t]{17}{*}{ Asteraceae } & Ageratum corymbosum Zuccagni ex Pers. & & $x$ & & & & & Herb & 72 \\
\hline & Bidens pilosa $\mathrm{L}$. & $x$ & $x$ & & & $x$ & $x$ & Herb & 230 \\
\hline & Critonia quadrangularis (DC.) R.M.King \& H.Rob. & & & & $x$ & & & Shrub & 3 \\
\hline & Cyanthillium cinereum (L.) H.Rob. & & $x$ & & & & $x$ & Herb & 7 \\
\hline & Chromolaena odorata (L.) R.M.King \& H.Rob. & & $x$ & & & & & Herb & 9 \\
\hline & Eclipta prostrata (L.) L. & & & & & & $x$ & Herb & 4 \\
\hline & Erigeron procumbens (Houst. ex Mill.) G.L.Nesom & $x$ & $x$ & & & & & Herb & 10 \\
\hline & Florestina pedata (Cav.) Cass. & $x$ & $x$ & & & $x$ & & Herb & 130 \\
\hline & Melampodium americanum L. & & $x$ & & & $x$ & & Herb & 36 \\
\hline & Mikania micrantha Kunth & & & & & & $x$ & Vine & 7 \\
\hline & Neurolaena lobata (L.) Cass.R.Br. $\left({ }^{*}\right)$ & & & & & & $x$ & Shrub & 6 \\
\hline & Palafoxia lindenii A.Gray & & $x$ & & & & & Herb & 82 \\
\hline & Pectis saturejoides (Mill.) Sch. Bip. & $x$ & $x$ & & & $x$ & & Herb & 70 \\
\hline & Pluchea odorata (L.) Cass. & & $x$ & & & & & Shrub & 4 \\
\hline & Porophyllum punctatum S.F.Blake & $x$ & $x$ & & & & & Herb & 111 \\
\hline & Tridax procumbens (L.) L. & & $x$ & & & & & Herb & 1 \\
\hline & Trixis inula Crantz & $x$ & $x$ & $x$ & & & & Herb & 9 \\
\hline Bignoniaceae & Tecoma stans (L.) Kunth & $x$ & $x$ & & & & & Shrub & 6 \\
\hline Bombacaceae & Pachira aquatica Aubl. & & & & & & $x$ & Tree & 12 \\
\hline \multirow[t]{4}{*}{ Boraginaceae } & Cordia spinescens L. & & $x$ & & & & $x$ & Shrub & 2 \\
\hline & Cordia polycephala (Lam.) I.M.Johnst. & $x$ & & & & & & Shrub & 3 \\
\hline & Tournefortia hirsutissima L. & $x$ & & & & & & Herb & 3 \\
\hline & Tournefortia volubilis L. & & & & & & $x$ & Herb & 3 \\
\hline \multirow[t]{2}{*}{ Bromeliaceae } & Tillandsia paucifolia Baker utriculata L. $\left({ }^{*}\right)$ & $x$ & & & & $x$ & & Herb & 1 \\
\hline & Tillandsia paucifolia Baker utriculata L. $(*)$ & $x$ & & & & $x$ & & Herb & 60 \\
\hline \multirow[t]{2}{*}{ Burseraceae } & Bursera fagaroides (Kunth) Engl. & & & & & $x$ & & Tree & 22 \\
\hline & Bursera simaruba (L.) Sarg. & $x$ & & $x$ & & $x$ & & Tree & 29 \\
\hline Cactaceae & Opuntia stricta (Haw.) Haw. & $x$ & & & & $x$ & & Shrub & 133 \\
\hline \multirow[t]{2}{*}{ Caesalpiniaceae } & Caesalpinia bonduc (L.) Roxb. & & $x$ & & & & & Shrub & 36 \\
\hline & Chamaecrista chamaecristoides Greene & $x$ & $x$ & & & & & Shrub & 70 \\
\hline \multirow[t]{2}{*}{ Capparaceae } & Capparidastrum frondosum (Jacq.) Cornejo \& Iltis $(*)$ & & & & $x$ & & & Shrub & 3 \\
\hline & Cynophalla flexuosa (L.) J. Presl & & $x$ & & & & & Shrub & 1 \\
\hline
\end{tabular}


Table 1. Continuation.

\begin{tabular}{|c|c|c|c|c|c|c|c|c|c|}
\hline \multirow[t]{2}{*}{ FAMILY } & \multirow[t]{2}{*}{ SPECIES } & \multicolumn{6}{|c|}{ ENVIRONMENT } & \multirow{2}{*}{$\begin{array}{l}\text { BIOL. } \\
\text { FORM }\end{array}$} & \multirow[t]{2}{*}{ FREQUENCY } \\
\hline & & CDS & PDV & TSF-1 & TSF-2 & TDF & TDF-W & & \\
\hline Caricaceae & Carica papaya L. & & & & & & $x$ & Tree & 2 \\
\hline \multirow[t]{3}{*}{ Celastraceae } & Crossopetalum gaumeri (Loes.) Lundell $(*)$ & $x$ & & $x$ & & $x$ & & Shrub & 12 \\
\hline & Crossopetalum uragoga Kuntze & $x$ & & $x$ & $x$ & $x$ & & Shrub & 149 \\
\hline & Elaeodendron laneanum A.H. Moore & & & & & $x$ & & Tree & 3 \\
\hline \multirow[t]{2}{*}{ Combretaceae } & Conocarpus erectus $\mathrm{L}$. & & $x$ & & & & & Tree & 13 \\
\hline & Terminalia catappa L. & & & & & & $x$ & Tree & 1 \\
\hline Commelinaceae & Commelina erecta L. & $x$ & $x$ & & & $x$ & & Herb & 235 \\
\hline Connaraceae & Rourea glabra Kunth & & & & $x$ & $x$ & & Vine & 10 \\
\hline \multirow[t]{4}{*}{ Convolvulaceae } & Ipomoea aquatica Forssk. & & & & & & $x$ & Vine & 6 \\
\hline & Ipomoea pes-caprae (L.) Sweet & & $x$ & & & & & Vine & 48 \\
\hline & Ipomoea stolonifera (Cirillo) J.F. Gmel. & & $x$ & & & & & Vine & 78 \\
\hline & Merremia umbellata (L.) Hallier f. & & & & & & $x$ & Vine & 3 \\
\hline \multirow[t]{2}{*}{ Cucurbitaceae } & Melothria pendula L. & $x$ & & & & & & Vine & 2 \\
\hline & Momordica charantia L. & & $x$ & & & & & Vine & 1 \\
\hline Chrysobalanaceae & Chrysobalanus icaco L. & $x$ & & & & $x$ & & Tree & 38 \\
\hline Ebenaceae & Diospyros verae-crucis (Standl.) Standl. & $x$ & & & & & & Tree & 5 \\
\hline Erythroxylaceae & Erythroxylum havanense Jacq. & & & & & $x$ & & Tree & 1 \\
\hline \multirow[t]{5}{*}{ Euphorbiaceae } & Adelia barbinervis Cham. \& Schltdl. \& Cham. & & & & & & $x$ & Shrub & 1 \\
\hline & Cnidoscolus herbaceus (L.) I.M. Johnst. & $x$ & & & & $x$ & & Herb & 42 \\
\hline & Croton punctatus Lour. & & $x$ & & & & & Shrub & 20 \\
\hline & Euphorbia heterophylla L. & & & & & & $x$ & Herb & 2 \\
\hline & Manihot aesculifolia (Kunth) Pohl & & $x$ & & & $x$ & & Shrub & 12 \\
\hline \multirow[t]{16}{*}{ Fabaceae } & Calopogonium caeruleum (Benth.) C. WrightHemsl. & & & & & & $x$ & Herb & 7 \\
\hline & Canavalia rosea (Sw.) DC. & $x$ & $x$ & & & & & Vine & 65 \\
\hline & Centrosema virginianum (L.) Benth. & $x$ & & & & & & Vine & 18 \\
\hline & Crotalaria incana L. & $x$ & $x$ & & & & & Shrub & 101 \\
\hline & Dalbergia brownei (Jacq.) Schinz & & $x$ & & & & $x$ & Shrub & 46 \\
\hline & Desmodium adscendens (Sw.) DC. & & & & & & $x$ & Herb & 4 \\
\hline & Desmodium scorpiurus (Sw.) Poir. $\left(^{*}\right)$ & $x$ & $x$ & & & & $x$ & Herb & 4 \\
\hline & Gliricidia sepium (Jacq.) Kunth ex Walp. & & $x$ & & & & & Tree & 5 \\
\hline & Indigofera miniata Ortega & $x$ & & & & $x$ & & Herb & 6 \\
\hline & Lonchocarpus fuscopurpureus Brandegee $\left(^{*}\right)$ & & & & & $x$ & & Tree & 1 \\
\hline & Macroptilium atropurpureum (L.) Urb. & $x$ & $x$ & & & $x$ & & Vine & 172 \\
\hline & Rhynchosia americana (Mill.) Metz & $x$ & & & & & $x$ & Vine & 7 \\
\hline & Rhynchosia minima (L.) DC. & & & & & & $x$ & Vine & 2 \\
\hline & Tephrosia cinerea (L.) Pers. & $x$ & & & & $x$ & & Herb & 16 \\
\hline & $\begin{array}{l}\text { Vigna adenantha (G. Mey.) Maréchal, Mascherpa } \\
\& \text { Stainier }\left({ }^{*}\right)\end{array}$ & & $x$ & & & & & Vine & 4 \\
\hline & Vigna vexillata (L.) A. Rich. & & & & & & $x$ & Vine & 7 \\
\hline Flacourtiaceae & Casearia corymbosa Kunth & $x$ & & & & & & shrub & 7 \\
\hline \multirow[t]{2}{*}{ Lamiaceae } & Aegiphila deppeana Steud. & $x$ & & & & & $x$ & vine & 8 \\
\hline & Callicarpa acuminata Kunth & $x$ & & & & & & shrub & 2 \\
\hline \multirow[t]{2}{*}{ Leguminosae } & Diphysa americana (Mill.) M. Sousa & $\mathrm{X}$ & $\mathrm{X}$ & & & & & Tree & 2 \\
\hline & Mucuna pruriens (L.) DC. & & & & & & $x$ & Vine & 7 \\
\hline Loranthaceae & Psittacanthus schiedeanus (Schltdl. \& Cham.) Blume & $x$ & & & & $x$ & & shrub & 8 \\
\hline Malpighiaceae & Malpighia glabra L. & & & $x$ & & & & Shrub & 1 \\
\hline \multirow[t]{6}{*}{ Malvaceae } & Anoda cristata (L.) Schltdl. & & & & & & $x$ & Herb & 7 \\
\hline & Herissantia crispa (L.) Brizicky & & & & & & $x$ & Herb & 1 \\
\hline & Kosteletzkya depressa (L.) O.J. Blanch., Fryxell & & & & & & $x$ & Herb & 1 \\
\hline & \& D.M. Bates & & & & & & & & \\
\hline & Malachra capitata (L.) L. & & & & & & $x$ & Herb & 1 \\
\hline & Sida acuta Burm. f. & $x$ & & & & & $\mathrm{x}$ & Herb & 10 \\
\hline Marantaceae & Thalia geniculata L. & & & & & & $x$ & Herb & 5 \\
\hline
\end{tabular}


Table 1. Continuation.

\begin{tabular}{|c|c|c|c|c|c|c|c|c|c|}
\hline \multirow[t]{2}{*}{ FAMILY } & \multirow[t]{2}{*}{ SPECIES } & \multicolumn{6}{|c|}{ ENVIRONMENT } & \multirow{2}{*}{$\begin{array}{l}\text { BIOL. } \\
\text { FORM }\end{array}$} & \multirow[t]{2}{*}{ FREQUENCY } \\
\hline & & CDS & PDV & TSF-1 & TSF-2 & TDF & TDF-W & & \\
\hline \multirow[t]{5}{*}{ Mimosaceae } & Acacia cornigera (L.) Willd. & & & & & & $x$ & Shrub & 8 \\
\hline & Calliandra eriophylla Benth. $(*)$ & & & & & $x$ & & Shrub & 2 \\
\hline & $\begin{array}{l}\text { Lysiloma divaricatum (Jacq.) J.F. Macbr. Hook. } \\
\text { \& B.D.Jacks. }\end{array}$ & & & & & $x$ & & Tree & 27 \\
\hline & Mimosa tricephala Cham. \& Schltdl. \& Cham. & $x$ & & & & & & Shrub & 10 \\
\hline & Neptunia plena (L.) Benth. & & & & & & $x$ & Herb & 2 \\
\hline Moraceae & Brosimum alicastrum Sw. & $x$ & & & $x$ & & & Tree & 2 \\
\hline \multirow[t]{2}{*}{ Myrtaceae } & Eugenia acapulcensis Steud. & $x$ & & & & & & shrub & 12 \\
\hline & Psidium guajava L. & & & & & & $x$ & tree & 3 \\
\hline Nymphaeaceae & Nymphaea ampla (Salisb.) DC. & & & & & & $x$ & herb & 7 \\
\hline \multirow{2}{*}{ Onagraceae } & Ludwigia leptocarpa (Nutt.) H. Hara & & & & & & $x$ & herb & 2 \\
\hline & Ludwigia octovalvis (Jacq.) P.H. Raven & & $x$ & & & & $x$ & herb & 43 \\
\hline Orchidaceae & $\begin{array}{l}\text { Trichocentrum luridum (Lindl.) M.W.Chase } \\
\& \text { N.H.Williams }(*)\end{array}$ & & & & $x$ & & & herb & 1 \\
\hline Oxalidaceae & Oxalis frutescens $\mathrm{L}$. & & & & & $x$ & & herb & 7 \\
\hline \multirow[t]{2}{*}{ Passifloraceae } & Passiflora holosericea L. & $x$ & & & & & & vine & 2 \\
\hline & Passiflora suberosa L. & & & & & & $x$ & vine & 1 \\
\hline \multirow[t]{2}{*}{ Phytolaccaceae } & Petiveria alliacea L. & & & $x$ & $x$ & & & herb & 15 \\
\hline & Rivina humilis L. & & & & $x$ & & $x$ & herb & 4 \\
\hline \multirow{2}{*}{ Piperaceae } & Piper amalago L. & & & & & & $x$ & shrub & 3 \\
\hline & Piper nitidum Sw. & & & & & & $x$ & shrub & 17 \\
\hline \multirow[t]{2}{*}{ Polygonaceae } & Coccoloba humboldtii Meisn. & & $x$ & & & $x$ & & tree & 19 \\
\hline & Coccoloba liebmannii Lindau & & & & $x$ & & & tree & 1 \\
\hline Pontederiaceae & Pontederia sagittata C. PresI & & & & & & $x$ & herb & 82 \\
\hline Rafflesiaceae & $\begin{array}{l}\text { Bdallophytum americanum (R. Br.) Eichler } \\
\text { ex SolmsHarms }\end{array}$ & & & & $x$ & & & Herb & 1 \\
\hline Ranunculaceae & Clematis grossa Benth. & & & & & & $x$ & vine & 2 \\
\hline Rhamnaceae & Rhamnus humboldtiana Willd. ex Schult. & & & & & $x$ & & tree & 3 \\
\hline \multirow[t]{5}{*}{ Rubiaceae } & Chiococca coriacea M. Martens \& Galeotti & $x$ & $x$ & & & $x$ & & shrub & 65 \\
\hline & Diodia teres Walter & & & & & $x$ & & herb & 8 \\
\hline & Psychotria erythrocarpa Schltdl. & & & $x$ & & $x$ & $x$ & shrub & 21 \\
\hline & Randia aculeata L. var. dasyclada Steyerm. & $x$ & $x$ & & & $x$ & & shrub & 142 \\
\hline & Spermacoce assurgens Ruiz \& Pav. & & & & & & $x$ & herb & 1 \\
\hline \multirow[t]{2}{*}{ Sapindaceae } & Cardiospermum halicacabum L. & $x$ & & & & & & vine & 10 \\
\hline & Paullinia tomentosa Jacq. & $x$ & $x$ & & & $x$ & & vine & 22 \\
\hline \multirow[t]{2}{*}{ Scrophulariaceae } & Bacopa monnieri (L.) Wettst. & & & & & & $x$ & herb & 4 \\
\hline & Russelia sarmentosa Jacq. & & & & & $x$ & & herb & 34 \\
\hline \multirow[t]{2}{*}{ Solanaceae } & Solanum diphyllum L. & & & & & & $x$ & shrub & 2 \\
\hline & Solanum rudepannum Solanum rudepannum Dunal & & & & & & $x$ & shrub & 1 \\
\hline Sterculiaceae & Waltheria indica $\mathrm{L}$. & $x$ & $x$ & & & $x$ & $x$ & Herb & 60 \\
\hline Theophrastaceae & Jacquinia macrocarpa Cav. & & & $x$ & $x$ & & & tree & 9 \\
\hline Tiliaceae & Muntingia calabura L. & & & & & & $x$ & Tree & 2 \\
\hline & Triumfetta lappula L. & & & & $x$ & & & Shrub & 2 \\
\hline Turneraceae & Turnera diffusa Willd. & $x$ & & & & $x$ & & herb & 37 \\
\hline & Turnera ulmifolia L. & $x$ & $x$ & & & & $x$ & herb & 99 \\
\hline Verbenaceae & Lantana camara L. & $x$ & $x$ & & & $x$ & & shrub & 79 \\
\hline & Lippia graveolens Kunth & & & & & $x$ & & herb & 4 \\
\hline & Petrea volubilis L. & & & & $x$ & & & vine & 3 \\
\hline & Phyla nodiflora (L.) Greene & & $x$ & & & & $x$ & herb & 32 \\
\hline & Priva lappulacea (L.) Pers. & & & & & & $x$ & herb & 2 \\
\hline & Tamonea curassavica (L.) Pers. & & & & & $x$ & & herb & 30 \\
\hline Viscaceae & Phoradendron quadrangulare (Kunth) Griseb.Trel. & $x$ & $x$ & & & & & herb & 5 \\
\hline Vitaceae & Vitis tiliifolia Humb. \& Bonpl. ex Schult. & & $x$ & & & & $x$ & vine & 4 \\
\hline
\end{tabular}


Table 2. Chao-Jaccard Similarity Index for the six vegetation types. The bold numbers belongs to highest values of similarity.

\begin{tabular}{lcccccc}
\hline & CDS & PDV & TSF-1 & TSF-2 & TDF & TDF-W \\
\hline CDS & 1 & $\mathbf{0 . 4 7 9}$ & 0.06 & 0.04 & $\mathbf{0 . 5 1 8}$ & 0.036 \\
PDV & 1 & 0.003 & 0 & 0.205 & 0.075 \\
TSF-1 & & 1 & 0.444 & 0.145 & 0.081 \\
TSF-2 & & & & 1 & 0.004 \\
TDF & & & & & 1 \\
TDF-W & & & & & \\
\hline
\end{tabular}
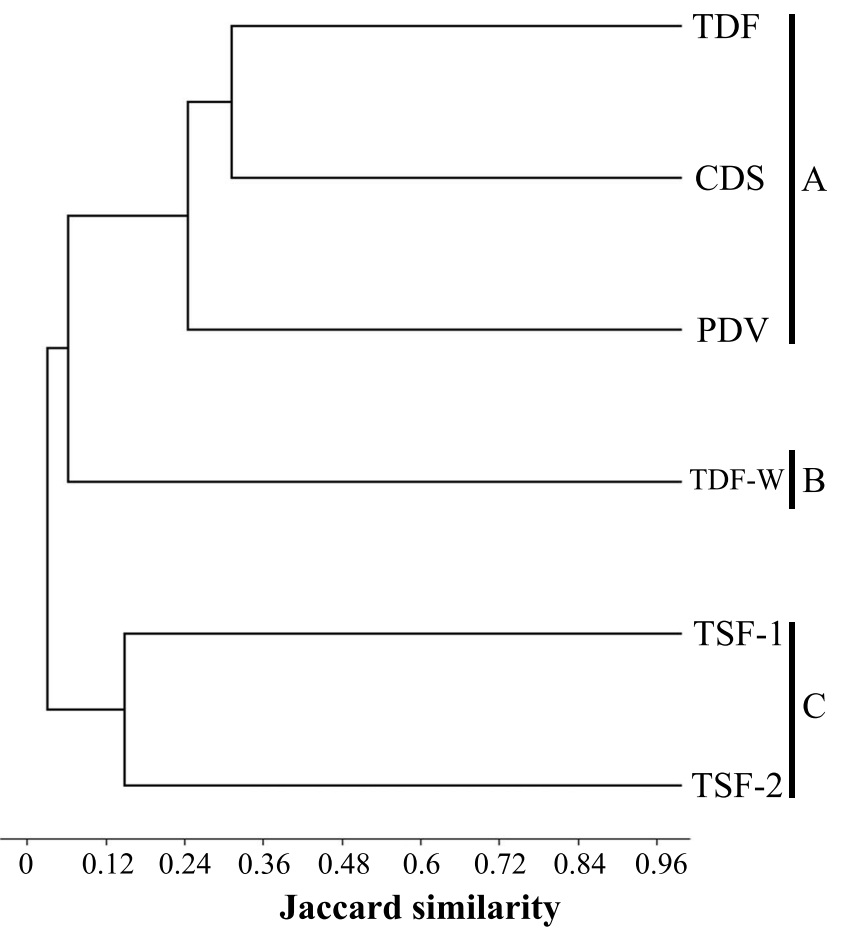

Figure 4. Clustering analyses using Jaccard Similarity Index. $\mathrm{TDF}=$ tropical deciduous forest, $\mathrm{CDS}=$ coastal dune scrub, $\mathrm{PDV}=$ pioneer dune vegetation, TDF-W = tropical deciduous flood forest with wetland, TSF-1 = tropical sub-deciduous forest in young soil, and TSF-2 $=$ tropical sub-deciduous forest in old soil.

Biological forms. Considering the six vegetation types, the herbs contribute with $40.41 \%$ of species, followed by shrubs $(23.97 \%)$, vines-lianas (18.49\%), and trees (17.12\%). Moreover, herbs were represented mostly by Asteraceae, whereas shrubs, vines-lianas, and trees were well represented by Fabaceae. On other hand, the herbs were the most represented biological forms followed by shrubs in coastal dune scrub, pioneer dune vegetation, and tropical deciduous forest. Shrubs and herbs were the most important biological form in tropical sub-deciduous forest in old soil. Moreover herbs were highly represented as main plants in the tropical deciduous flood forest with wetland; however, this site showed that vines-lianas were well represented as well.
Flowering and fruiting display. Overall, our results showed that species with the highest frequency of occurrence in these phenological stages were: Commelina erecta, Bidens pilosa, Macroptilium atropurpureum, Crossopetalum uragoga (Table 1). On other hand, and some species like C. erecta, B. pilosa, Palafoxia lindenii, M. atropurpureum, and C. uragoga had a flower display and fruit setting in long periods through year, while, Randia aculeata var. dasyclada, Opuntia stricta, and Florestina pedata had a flower display in some months and fruit setting on almost months posterior to flowering.

In addition, the species that had high frequencies also we were recorded in three or four vegetation types, like Bidens pilosa and Crossopetalum uragoga which occurred in four, followed by Commelina erecta, Macroptilium atropurpureum, Randia aculeata var. dasyclada, and Florestina pedata in three vegetation types. The other species occurred in less than three vegetation types and had low frequencies (Table 1). We also classified the plant species based on floral symmetry and we observed that plant species with actinomorphic flowers had higher number of species $(67 \%)$ than those plants species with zygomorphic $(25 \%)$ and asymmetric $(8$ $\%)$ flowers. Asteraceae showed the highest number of actinomorphic species, while Fabaceae, Mimosaceae and Caesalpiniaceae had more zygomorphic and asymmetric flowers species.

\section{Discussion}

In this study we recorded 147 plant species, that represent $53.4 \%$ of all families, genera (28.1\%), and species (17.5\%) registered in La Mancha by Castillo-Campos and TraviesoBello (2006), also this represent $1.85 \%$ of total flora of Veracruz State (Dirzo and Gómez, 1996; Castillo-Campos et al., 2011). Inside of Veracruz State the great floristic diversity is distributed in 19 vegetation types (Miranda and Hernández-X., 1963; Castillo-Campos et al., 2011). In other areas, like Los Tuxtlas Biosphere Reserve (329,941 ha) have been reported 22 vegetation types, containing 3,356 species and 212 families (Castillo-Campos and Laborde, 2004); in La Sierra Cruz Tetela 569 species were reported that belong to 110 families, which 22 are introduced in crop fields in three vegetation types (Castillo-Campos et al., 2003), while at the present study we recorded plant species only in six vegeta- 
tion types, inside a very small area (83.29 ha), focusing only at flowering and/or fruiting species, discarding those without reproductive events, thus, our study is a small representation of all the plant diversity at the state scale. Despite this we found nearly half of species recorded in previous studies in the same place that used specific methods for vegetation analyses (Castillo-Campos and Travieso-Bello, 2006).

Other important findings are the nine new species that have not been reported previously in the existent inventories in La Mancha. This is important to be highlighted because new species could be present at these environments, as well as determining if these species are ruderals or potentially become invasive species. On the other hand, species like Crossopetalum gaumeri, Calliandra eriophylla, and Lonchocarpus fuscopurpureus have been registered and accessed as herbarium specimens (XAL-Herbarium), but have not been recorded in floristic studies.

Several studies in Veracruz found that the families with largest number of species were Fabaceae and Asteraceae mainly (Castillo and Moreno-Casasola, 1998; Castillo-Campos et al., 2003; Castillo-Campos and Travieso-Bello, 2006; Arroyo-Rodríguez et al., 2009), and in other studies the second most represented family was Asteraceae (Castillo and Moreno-Casasola, 1998; Castillo-Campos and Travieso-Bello, 2006; Espinosa-Jiménez et al., 2014), similarly, our findings showed this pattern. At the present study a concentration of species richness in few families was found: $27 \%$ of all recorded species were comprised in only three families. This pattern is concordant with other floristic studies, i.e. in Los Tuxtlas Biosphere Reserve on 45 rainforest fragments $21 \%$ of all identified plant species, were concentrated within only four families (Arroyo-Rodríguez et al., 2009); also in coastal dune systems in central Veracruz, more than half species are represented in a small number of families (16 of 91 families) (Castillo and Moreno-Casasola, 1998).

Considering the species richness in different vegetation types and comparing it with the total of species reported for Veracruz (7,855 species) (Castillo-Campos et al., 2011), we found that tropical flood deciduous forest with wetland represent $0.75 \%$ of plant species in the state, while coastal dune vegetation (considering pioneer dune vegetation and dune scrub for this research) represent $0.94 \%$, and for tropical deciduous forest only $0.52 \%$. Particularly, for wetlands at La Mancha, Infante-Mata et al. (2011) reported 12 species while in present study we found 59 species. On other hand, in contrast with the list of Castillo-Campos and Travieso-Bello (2006), our more-local survey the local representation of this survey; the tropical flood deciduous forest with wetland represent $62.1 \%$ of total plant species, coastal dune vegetation represents $35.1 \%$, and tropical deciduous forest a $9.51 \%$. Thus, the floristic survey provided at the present study is truly representative of the sampled vegetation types.

Moreover, Castillo-Campos and Travieso-Bello (2006) found that the floristic communities of La Mancha that share a large number of species and have a higher similarity were the coastal dune vegetation, deciduous forest, and tropical sub-deciduous forest. These results were similar to those observed in our study, since the cluster analysis according to the identity and frequency of each species as well as the analysis of Chao-Jaccard, showed higher similarity between coastal dune scrub, tropical deciduous forest, and pioneer dune vegetation. This pattern was also observed for the analysis of floristic diversity associated with extrafloral nectaries in La Mancha, where the vegetation types with highest similarity values (in number of shared species) were coastal dune scrub and pioneer dune vegetation (DiazCastelazo et al., 2004). The pattern showed at the present study points out that coastal dune scrub, tropical deciduous forest and pioneer dune vegetation in La Mancha, shared a large number of species and similar frequencies, making them more similar to each other.

In general, another important aspect within plant communities is the diversity of biological forms, that, depending of the vegetation type, are represented by trees, shrubs, vines-lianas, and herbs, and in some cases are considered as functional groups (Ribeiro et al., 1999). Frequently, herbs represent the main number of species, followed by shrubs, trees and vines-lianas within plant communities (Dirzo and, Gómez, 1996; Castillo-Campos et al., 2003; CastilloCampos et al., 2011), and in some studies shrubs, trees, and vines-lianas can change in the order of importance in which they are represented (Castillo-Campos et al., 2003; Castillo-Campos and Laborde, 2004; Ponce-Vargas et al., 2006; Espinosa-Jiménez et al., 2014). Also this pattern was observed in the plant community of La Mancha (Castillo and Moreno-Casasola, 1998; Castillo-Campos and TraviesoBello, 2006), and similar to observations in our study, herbs were the most importantly represented and only changing the order of trees and vines-lianas. In addition, our results were consistent with reported in the literature for Asteraceae as a family with most contribution to herbs, while Fabaceae, Mimosaceae, and Caesalpiniaceae have an important contribution to the tree, shrub, and vine-liana biological forms, similarly to reports by Espinosa-Jiménez et al., (2014). Herbs also were well represented on all vegetation types in this study followed by shrubs, and trees, while vines-lianas were only well represented for the tropical flood forest with wetland, in accordance to Castillo-Campos and TraviesoBello (2006). Thus, the more representative plant biological form for La Mancha at the present study was herbs and this biological form was mainly represented by Asteraceae, whereas the trees, shrubs, and vines-lianas were comprised in Fabaceae, Mimosaceae, and Caesalpiniaceae.

The frequency, density and abundance of plant species in different environments involving reproductive events (i.e. flowering and fruiting) is not common in floristic studies; this information was also lacking for plant communities in La Mancha, where there are no studies considering these 
variables in different plant species. Recently Infante-Mata et al. (2011) found (using absolute densities) that Pachira aquatica, Ficus insipida subsp. insipida, and Annona glabra are the most common species of trees and the vines-lianas Dalbergia brownei, Hippocratea celastroides, and Rhabdadenia biflora in swamp of La Mancha. Our study reported also $P$. aquatica, A. glabra, and D. brownei in tropical deciduous flood forest with wetland, however, from our perspective of flowering and fruiting plants, these three species showed very low frequencies. On the other hand, we observed that plant species with high frequency of occurrence (as Bidens pilosa and Crossopetalum uragoga) occurred in four vegetation types, while, Commelina erecta, Macroptilium atropurpureum, Randia aculeata var. dasyclada, and Florestina pedata occurred in three vegetation types, in accordance to the information of the existing plant checklist (Castillo-Campos and Travieso-Bello, 2006), because these species occur in the same environments in both studies.

In summary, this is the first study that evaluates the frequency-occurrence of plants flowering or fruiting in six vegetation types simultaneously through one year, comprising dry, rainy, and winter cold-front seasons. The relevance and novelty of our study is that it shows the diversity of plant species in different environments, but more importantly is to show the frequency of each species based on reproductive events. This information can be used in future research evaluating ecological and biological processes at community level or in plant-animal interactions, i.e. pollination and seed dispersal.

\section{Acknowledgements}

This study was developed as part of doctoral studies of CAMA performed with scholarship number 230073 at the doctorate program of INECOL; financial support for fieldwork was provided by project 2003011143 to CDC. We give special thanks to Postgraduate Secretary of INECOL and Multitrophic Interactions Department (Red de Interacciones Multitróficas) for the administrative facilities to perform this study. Also we give special thanks to Elisa Zaragoza for map elaboration, and fieldwork assistance; also we thank J. Luis Sánchez, and Rosalba Quintana for their valuable help in the field; we are grateful to Gonzalo Castillo-Campos, Miguel Cházaro, Sergio Avendaño, Santiago Sinaca, Carlos Durán, José G. García-Franco, Miguel Domínguez, Diana Vázquez, Antonio López-Carretero, and Jonás Morales for their help with taxonomic identification. In addition, we thanks to the anonymous reviewers for help to improve the clarity of this manuscript.

\section{Literature cited}

Álvarez-Molina L.L., Martínez M.L., Lithgow D., MendozaGonzález G., Flores P., Ortíz-García S. and Moreno-Casasola P.
2013. Biological flora of coastal dunes and wetlands: Palafoxia lindenii A. Gray. Journal of Coastal Research 29:680-693.

Arceo-Gómez G., Martínez M.L., Parra-Tabla V. and García-Franco J.G. 2012. Floral and reproductive biology of the Mexican endemic Chamaecrista chamaecristoides (Fabaceae). Journal of the Torrey Botanical Society 139:260-269.

Arroyo-Rodríguez V., Dunn J.C., Benítez-Malvido J. and Mandujano S. 2009. Angiosperms, Los Tuxtlas Biosphere Reserve, Veracruz, Mexico. Check List 5:787-799.

Benítez-Malvido J. 1998. Impact of forest fragmentation on seedling abundance in a tropical rain forest. Conservation Biology 12:380-389.

Benítez-Malvido J., García-Guzman G. and Kossmann-Ferraz I.D. 1999. Leaf-fungal incidence and herbivory on tree seedlings in tropical rainforest fragments: an experimental study. Biological Conservation 91:143-150.

Castillo S. and Carabias J. 1982. Ecología de la vegetación de dunas costeras: fenología. Biotica 7:551-569.

Castillo S.A. and Moreno-Casasola P. 1998. Análisis de la flora de dunas costera del litoral Atlántico de México. Acta Botanica Mexicana 45:55-80.

Castillo-Campos G., Robles-González R. and Medina-Abreo M.E. 2003. Flora y vegetación de la Sierra Cruz Tetela, Veracruz, Mexico. Polibotanica 15:41-87.

Castillo-Campos G. and Laborde J.D. 2004. La vegetación. In: Guevara S., Laborde J., and Sánchez-Ríos G. Eds. Los Tuxtlas. El Paisaje de la Sierra, pp. 231-270, Instituto de Ecología, A.C. y la Unión Europea, Xalapa.

Castillo-Campos G. and Medina-Abreo M.E. 2005. Árboles y Arbustos de la Reserva Natural de La Mancha, Veracruz. Manual para la Identificación de las Especies. Instituto de Ecología, A.C., Xalapa.

Castillo-Campos G. 2006. Las Selvas. In: Moreno-Casasola P. Ed. Entornos Veracruzanos: la Costa de La Mancha, pp. 221-229, Instituto de Ecología A.C., Xalapa.

Castillo-Campos G. and Travieso-Bello A.C. 2006. La flora. In: Moreno-Casasola P. Ed. Entornos Veracruzanos: La Costa de La Mancha, pp. 171-204, Instituto de Ecología A.C., Xalapa.

Castillo-Campos G., Avendaño-Reyes S. and Medina-Abreo M.E. 2011. Flora y vegetación. In: CONABIO. La Biodiversidad en Veracruz: Estudio de Estado, Volumen I,, pp. 163-179, Comisión Nacional para el Conocimiento y Uso de la Biodiversidad, Gobierno del Estado de Veracruz, Universidad Veracruzana, Instituto de Ecología, A.C., México D.F.

Ceballos G. 2007. Conservation priorities for mammals in megadiverse Mexico: the efficiency of reserve networks. Ecological Applications 17:569-578.

Chacoff N.P., Morales J.M. and Vaquera M. del P. 2004. Efectos de la fragmentación sobre la aborción y depredación de semillas en el Chaco Serrano. Biotropica 36:109-117.

Chao A., Chazdon R.L., Colwell R.K. and Shen T.-J. 2005. A new statistical approach for assessing compositional similarity based on incidence and abundance data. Ecology Letters 8:148-159.

Colwell R.K. 2013. EstimateS: Statistical estimation of species richness and shared species from samples. Version 9.0.0. $<\mathrm{http}$ //viceroy.eeb.uconn.edu/estimates/index.html>

Cordeiro N.J. and Howe H.F. 2001. Low recruitment of trees dispersed by animals in African forest fragments. Conservation Biology 15:1733-1741.

Cuautle M. and Rico-Gray V. 2003. The effect of wasps and ants on 
the reproductive success of the extrafloral nectaried plant Turnera ulmifolia (Turneraceae). Functional Ecology 17:417-423.

Díaz-Castelazo C., Rico-Gray V., Oliveira P.S. and Cuautle M. 2004. Extrafloral nectary-mediated ant-plant interactions in the coastal vegetation of Veracruz, Mexico: Richness, occurrence, seasonality, and ant foraging patterns. Ecoscience 11:472-481.

Díaz-Castelazo C., Guimarães P.R.Jr., Jordano P., Thompson J.N., Marquis R.J. and Rico-Gray V. 2010. Changes of a mutualistic network over time: reanalysis over a 10-year period. Ecology 91:793-801.

Dirzo R. and Gómez G. 1996. Ritmos temporales de la investigación taxonómica de plantas vasculares en México y una estimación del número de especies conocidas. Annals of the Missouri Botanical Garden 83:396-403.

Dubroeucq D., Geissert D., Moreno P. and Millot G. 1992. Soil evolution and plant communities in coastal dunes near Veracruz, Mexico. Cahiers ORSTOM, Série Pédologie 27:237-250.

Espinosa-Jiménez J.A., López-Cruz A., Pérez-Farrera M.A. and López S. 2014. Inventario florístico de la cañada La ChaconaJuan Crispín y zonas adyacentes, depresión Central de Chiapas, México. Botanical Sciences 92:205-241.

Fragoso C. 1995. Propuesta de manejo de recursos y prioridades de investigación en el CICOLMA. Madera y bosques 1:61-63.

Franco-Gaona A., Llorente-Bousquets J. and Shapiro A.M. 1988. Abundancia relativa de Artrogeia rapae (L.), Pontiaprotodice (Boisd. \& Lec.) (Lepidoptera: Pieridae) evaluada mediante el método de Moore modificado por Pollard, en Xochimilco, D.F., México. Folia Entomológica Mexicana 76:107-128.

García-Franco J.G. 1996. Distribución de epífitas vasculares en matorrales costeros de Veracruz, México. Acta Botanica Mexicana 37:1-9.

García-Franco J.G. and Rico-Gray V. 1997a. Dispersión, viabilidad, germinación y banco de semillas de Bdallophyton bambusarum (Rafflesiaceae) en la costa de Veracruz, México. Revista de Biología Tropical 44/45:87-94.

García-Franco J.G. and Rico-Gray V. 1997b. Reproductive biology of the holoparasitic endophyte Bdallophyton bambusarum (Rafflesiaceae). Botanical Journal of the Linnean Society 123:237-247.

Geissert D. and Dubroeucq D. 1995. Influencia de la geomorfología en la evolución de suelos de dunas costeras en Veracruz, México. Investigaciones Geográficas Boletín Número Especial 3:37-51.

González-Medrano F. 2003. Las Comunidades Vegetales de México. Instituto Nacional de Ecología, México D.F.

González-Vanegas P.A. 2011. Visitantes florales y esfuerzo reproductivo de Pontederia sagittata C. Presl (Pontederiaceae) como indicadores del éxito de la restauración de un humedal en el centro de Veracruz, México. Tesis maestría, Instituto de Ecología, A.C. Xalapa, Veracruz, México. 60 pp.

Hammer Ø., Harper D.A.T., and Ryan P.D. 2001. PAST: Paleontological Statistics Software Package for Education and Data Analysis. Palaeontologia Electronica 4:4.

Hernández-Yáñez H., Lara-Rodríguez N., Díaz-Castelazo C., Dáttilo W. and Rico-Gray V. 2013. Understanding the complex structure of a plant-floral visitor network from different perspectives in Coastal Veracruz, Mexico. Sociobiology 60:329-336.

Infante-Mata D., Moreno-Casasola P., Madero-Vega C., CastilloCampos G. and Warner B.G. 2011. Floristic composition and soil characteristics of tropical freshwater forested wetlands of
Veracruz on the coastal plain of the Gulf of Mexico. Forest Ecology and Management 262:1514-1531.

Kavanagh T. and Kellman M. 1992. Seasonal pattern of fine root proliferation in a tropical dry forest. Biotropica 24:157-165.

Kellman M. 1990. Root proliferation in recent and weathered sandy soils from Veracruz, Mexico. Journal of Tropical Ecology 6:355-370.

Kellman M. and Roulet N. 1990. Nutrient flux and retention in a tropical sand-dune succession. Journal of Ecology 78:664-676.

Kellman M. and Delfosse B. 1993. Effect of the red land crab (Gecarcinus lateralis) on leaf litter in a tropical dry forest in Veracruz, Mexico. Journal of Tropical Ecology 9:55-65.

Martínez M.L. and Moreno-Casasola P. 1998. The biological flora of coastal dunes and wetlands: Chamaecrista chamaecristoides (Colladon) I. \& B. Journal of Coastal Research 14:162-174.

Mehltreter K. 2006. Leaf phenology of the climbing fern Lygodium venustum in a semideciduous lowland forest on the Gulf of Mexico. American Fern Journal 96:21-30.

Miranda F. and Hernández-X. E. 1963. Los tipos de vegetación de México y su clasificación. Boletín de la Sociedad Botánica de México 28:29-179.

Mittermeier R.A. and Mittermeier C.G. 1997. Megadiversity: Earth's Biologically Wealthiest Nations. CEMEX, México D.F.

Moreno-Casasola P. 1982. Ecología de la vegetación de dunas costeras: factores físicos. Biotica 7:577-602.

Moreno-Casasola P., Van der Maarel E., Castillo S., Huesca M.L., and Pisanty I. 1982. Ecología de la vegetación de dunas costeras: estructura y composición den el morro de La Mancha, Veracruz. I. Biotica 7:491-526.

Moreno-Casasola P. and Monroy R. 2006. Introducción. In: Moreno-Casasola P. Ed. Entornos Veracruzanos: La Costa de La Mancha, pp. 17-21, Instituto de Ecología A.C., Xalapa.

Moreno-Casasola P. and Travieso-Bello A.C. 2006. Las playas y dunas. In: Moreno-Casasola P. Ed. Entornos Veracruzanos: La Costa de La Mancha, pp. 205-220, Instituto de Ecología A.C., Xalapa.

Moreno-Casasola P., Cejudo-Espinosa E., Capistrán-Barradas A., Infante-Mata D., López-Rosas H., Castillo-Campos G., PalePale J. and Campos-Cascaredo A. 2010. Composición florística, diversidad y ecología de humedales herbáceos emergentes en la planicie costera central de Veracruz, México. Boletín de la Sociedad Botánica de México 87:29-50.

Neyra-González L. and Durand-Smith L. 1998. Biodiversidad. In: CONABIO. La Diversidad Biológica de México: Estudio de País, pp. 61-102, Comisión Nacional para el Conocimiento y Uso de la Biodiversidad, México D.F.

Novelo-Retana A. 1978. La vegetación de la estación biológica de El Morro de La Mancha, Veracruz. Biotica 3:9-23.

Ortiz-Pulido R. 1997. Actividades frugívoras de Tyrannus forficatus en un mosaico de vegetación durante la migración. Ornitologia Neotropical 8:237-239.

Peralta-Peláez L.A. and Moreno-Casasola P. 2009. Composición florística y diversidad de la vegetación de humedales en los lagos interdunarios de Veracruz. Boletín de la Sociedad Mexicana de Botánica 85:89-101.

Ponce-Vargas A., Luna-Vega I, Alcántara-Ayala O. and Ruiz-Jiménez C.A. 2006. Florística del bosque mesófilo de montaña de Monte Grande, Lolotla, Hidalgo, México. Revista Mexicana de Biodiversidad 77:177-190. 
Ribeiro J.E.L. d. S., Hopkins M.J.G., Vicentini A., Sothers C.A., Costa M.A. d. S., d. Brito J.M., Souza M.A. d., Martins L.H.P., Lohmann L.G., Assunção P.A.C.L., Pereira E. d. C., d. Silva C.F., Mesquita M.R. and Procópio L.C. 1999. Flora da Reserva Ducke: Guia de identificação das plantas vasculares de uma floresta de terra-firme na Amazônia Central. INPA-DFID, Manaus.

Rico-Gray V. 1993. Use of plant-derived food resources by ants in the dry tropical lowlands of coastal Veracruz, Mexico. Biotropica 25:301-315.

Rico-Gray V. and Castro G. 1996. Effect of ant-aphid interaction on the reproductive fitness of Paullinia fuscecens (Sapindaceae). The Southwestern Naturalist 41:434-440.

Rico-Gray V., García-Franco J.G., Palacios-Rios M., DíazCastelazo C., Parra-Tabla V. and Navarro J.A. 1998. Geographical and seasonal variation in the richness of ant-plant interactions in Mexico. Biotropica 30:190-200.

Rico-Gray V. and Oliveira P.S. 2007. The Ecology and Evolution of Ant-Plant Interactions. The University of Chicago Press, Chicago.

Rico-Gray V., Díaz-Castelazo C., Ramírez-Hernández A., Guimarães P.R.Jr. and Holland J.N. 2012. Abiotic factors shape temporal variation in the structure of a mutualistic ant-plant network. Arthropod-Plant Interactions 6:289-295.

Rodríguez-Morales D., Aguirre-Jaimes A., González-Vanegas P.A., López-Barrera F. and García-Franco J.G. 2013. Interacciones ecológicas en un humedal en restauración. Biodiversitas 106:11-16.

Rojas P., Fragoso C. and Mackay W.P. 2014. Ant communities along gradient of plant succession in Mexican tropical coastal dunes. Sociobiology 61:119-132.

Rzedowski J. 1991. Diversidad y orígenes de la flora fanerogámica de México. Acta Botanica Mexicana 14:3-21.

Rzedowski J. 1993. Diversity and origins of the phanerogamic flora of Mexico. In: Ramamoorthy T.P., Bye R., Lot A. and Fa J. Eds. Biological Diversity of Mexico: Origins and Distribution, pp. 129-144, Oxford University Press, New York.

Rzedowski J. 2006. Vegetación de México. Comisión Nacional para el Conocimiento y Uso de la Biodiversidad, México D.F.

Sánchez-Galván I.R., Díaz-Castelazo C. and Rico-Gray V. 2012.
Effect of hurricane Karl on a plant-ant network occurring in coastal Veracruz, Mexico. Journal of Tropical Ecology 28:603609.

Sarukhán J., Carabias J., Koleff P. and Urquiza-Haas T. 2012. Capital Natural de México: Acciones Estratégicas para su Valoración, Preservación y Recuperación. Comisión Nacional para el Conocimiento y uso de la Biodiversidad, México D.F.

Secretaría de la Convención de Ramsar. 2013. Manual de la Convención de Ramsar: Guía a la Convención sobre los Humedales (Ramsar, Irán, 1971). Secretaría de la Convención de Ramsar, Gland.

SEMARNAT [Secretaría del Medio Ambiente y Recursos Naturales]. 2010. Norma Oficial Mexicana NOM-059-SEMARNAT2010, Protección ambiental - Especies nativas de México de flora y fauna silvestres - Categorías de riesgo y especificaciones para su inclusión, exclusión o cambio - Lista de especies en riesgo. Diario Oficial de la Federación. 2da Sección, December $30,2010$.

Simpson E.H. 1949. Measurement of diversity. Nature 163:688688.

Simpson G.G. 1960. Notes on the measurement of faunal resemblance. American Journal of Science 258A:300-311.

Torres-Hernández L., Rico-Gray V., Castillo-Guevara C. and Vergara J.A. 2000. The effect of nectar-foraging ants and wasps on the reproductive fitness of Turnera ulmifolia (Turneraceae) in a coastal sand dune in México. Acta Zoológica Mexicana 81:13-21.

Travieso-Bello A.C. and Campos A. 2006. Los componentes del paisaje. In: Moreno-Casasola P. Ed. Entornos Veracruzanos: La Costa de La Mancha, pp. 139-150, Instituto de Ecología, A.C., Xalapa.

Turner I.M., Chua K.S., Ong J.S.Y., Soong B.C. and Tan H.T.W. 1996. A century of plant species loss from an isolated fragment of lowland tropical rain forest. Conservation Biology 10:12291244.

Wright S.J. and Duber H.C. 2001. Poachers and forest fragmentation alter seed dispersal, seed survival, and seedling recruitment in the palm Attalea butyracea, with implications for tropical tree diversity. Biotropica 33:583-595.

Received: October 9th, 2014

Accepted: December 12, 2014 